Utz von Wagner, Lukas Lentz

\title{
On artifact solutions of semi-analytic methods in nonlinear dynamics
}

Journal article | Accepted manuscript (Postprint)

This version is available at https://doi.org/10.14279/depositonce-9833

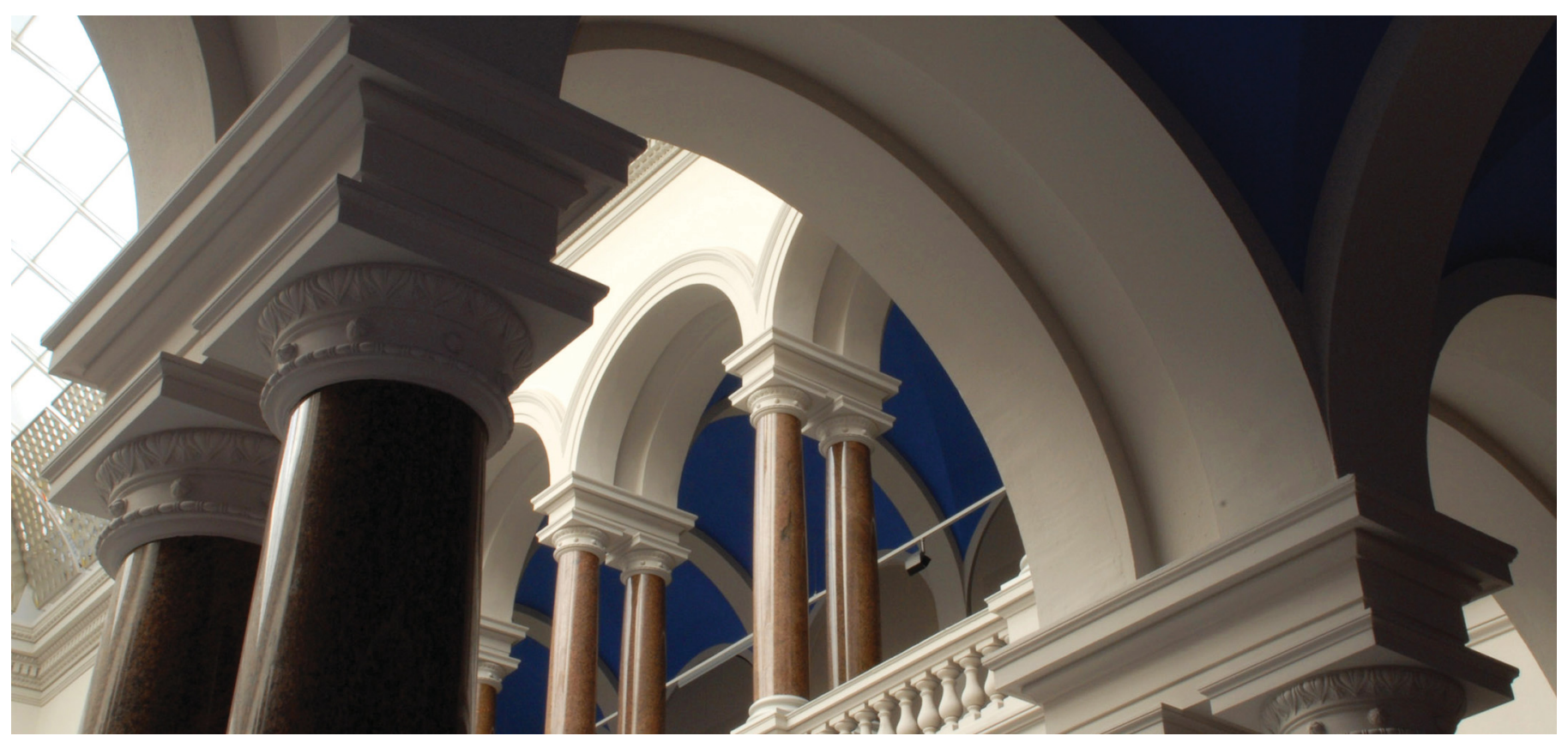

This is a post-peer-review, pre-copyedit version of an article published in Archive of Applied Mechanics.

The final authenticated version is available online at: http://dx.doi.org/10.1007/s00419-018-1397-3.

von Wagner, U., \& Lentz, L. (2018). On artifact solutions of semi-analytic methods in nonlinear dynamics. Archive of Applied Mechanics, 88(10), 1713-1724. https://doi.org/10.1007/s00419-018-1397-3 


\title{
On artifact solutions of semi-analytic methods in Nonlinear Dynamics
}

Received: date / Accepted: date

\begin{abstract}
Nonlinear dynamics is a topic of permanent interest in mechanics since decades. The authors recently published some results on a very classical topic, the dynamics of a softening Duffing oscillator under harmonic excitation focussing especially on low frequency excitation [1]. In this paper, it was shown that classical tools like Harmonic Balance and perturbation analysis may produce artificial solutions when applied without extra carefulness with respect to parameter ranges in the case of perturbation analysis or prior knowledge about the type of solution in case of Harmonic Balance. In the present paper these results are shortly summarized as they give the starting point for the additional investigations described herein. First, the method of slowly changing phase and amplitude is reviewed with respect to its capability for determining asymptotic stability of stationary solutions. It is shown, that this method can also produce artifact results when applied without extra carefulness. As next example an extended Duffing oscillator is investigated which shows, if Harmonic Balance is applied, "islands" of solutions. Using the error criterion in Harmonic Balance as described in [1] again artifact solutions can be identified.
\end{abstract}

Keywords Duffing oscillator, Harmonic Balance, perturbation analysis, artifact solutions

\section{Introduction}

Starting point of many fundamental investigations on nonlinear dynamics is the so-called Duffing oscillator named after the German engineer Georg Duffing (1861-1944), who investigated in his original work 1918 an oscillator with quadratic and cubic stiffness and linear viscous damping performing free or forced harmonic vibrations [2]. Nowadays the term "Duffing equation" is in general used for any nonlinear equation of motion including a cubic stiffness term. In that sense, we are investigating Duffing oscillators in the present paper starting from the classical one by giving a repetition of the methods used and the results obtained in [1] and adding afterwards additional nonlinearities including nonlinear damping and parametric excitation. A comprehensive book on scientific work done with respect to the Duffing oscillator is the one edited by Kovacic and Brennan [2]. In the present paper we investigate such problems using classical methods, especially the method of Harmonic Balance,

Utz von Wagner

Institut für Mechanik, Technische Universität Berlin, Einsteinufer 5, 10587 Berlin, Germany

Tel.: $+49 / 30 / 314-21169$

Fax: $+49 / 30 / 314-21173$

E-mail: utz.vonwagner@tu-berlin.de

Lukas Lentz

Institut für Mechanik, Technische Universität Berlin, Einsteinufer 5, 10587 Berlin, Germany

Tel.: +49/30/314-72415

Fax: +49/30/314-21173

E-mail: lukas.lentz@tu-berlin.de 
slowly changing phase and amplitude and perturbation analysis. All these methods are described in several textbooks as e.g. [3]. Especially the Harmonic Balance method gives, when applied to problems with a low number of degrees of freedom, results in a very rapid manner and often these results are at least qualitatively correct. In these cases they give a fast survey on the variety of solutions which is in nonlinear dynamics of essential importance, as multiple solutions can coexist. Therefore this method is very popular and still often applied. Compared to Harmonic Balance perturbation analysis is in general more difficult to apply, but often gives same or similar results. A big advantage of perturbation analysis compared to Harmonic Balance is, that there is no (or at least much less) need of prior knowledge of the structure of the solution. Perturbation analysis needs a careful checking of the order of the respective terms and is therefore often limited to certain parameter and initial condition ranges. On the other hand, if these points are carefully considered, results with high quality can be expected. Both methods are limited to stationary behavior and cannot be used for transient vibrations. As a simple method, that of slowly changing phase and amplitude can be applied in the transient case. This method will also be considered in this paper. Finally the method of multiple time scales as described broadly e.g. in [4] shall be mentioned combining the capabilities of perturbation analysis and slowly changing phase and amplitude, but very rapidly resulting in lengthy analytical calculations. Asymptotically stable solutions as a result of all mentioned methods can be verified using numerical integration in time, but variations of initial conditions must be performed, when multiple of such solutions coexist.

The present paper describes artifacts i.e. qualitatively wrong results, which can be produced by using the methods of Harmonic Balance, perturbation analysis and slowly changing phase and amplitudes for (modified) Duffing oscillators. An error criterion is used to identify these artifacts. Some of the mentioned points and methods were already investigated and applied in the paper [1] by the same authors for the case of a simple softening Duffing oscillator with harmonic excitation focusing on perturbation analysis and Harmonic Balance. In the present paper a section on artificial results which can be obtained by slowly changing phase and amplitude when applied for the aforementioned problem is included. In the following a modified Duffing oscillator is considered, which is capable to show "island"-like solutions when Harmonic Balance method is applied. These solutions are again investigated by using an error criterion.

\section{Artifact solution in classic softening Duffing oscillator}

Starting point of the following investigations will be the results described in the paper [1] by the same authors as the present one, which will therefore be described in a short summary in the following. Herein artifact and existing solutions of the classic Duffing oscillator containing damping and a softening cubic nonlinearity under harmonic excitation are described. The corresponding equation of motion reads as

$$
m \ddot{x}+d \dot{x}+c x+\alpha x^{3}=F_{0} \cos \Omega t
$$

with $m$ being the oscillator mass, $d$ the damping coefficient, $c$ the linear stiffness, $\alpha$ the coefficient of the nonlinear stiffness, $F_{0}$ the excitation force amplitude and $\Omega$ the circular excitation frequency. For the softening Duffing oscillator $\alpha$ is negative, while $m, d, c$ and $F_{0}$ are positive. This equation is rewritten by introducing the circular frequency of the undamped free linear vibrations $\omega_{0}^{2}=c / m$, the damping ratio $D=d /(2 \sqrt{\mathrm{cm}})$ and the dimensionless time $\tau=\omega_{0} t$ as

$$
x^{\prime \prime}(\tau)+2 D x^{\prime}(\tau)+x(\tau)+\varepsilon x^{3}(\tau)=f \cos (\eta \tau)
$$

with ()$^{\prime}=\mathrm{d}() / \mathrm{d} \tau, \varepsilon=\alpha /\left(m \omega_{0}^{2}\right), f=F_{0} /\left(m \omega_{0}^{2}\right)$ and $\eta=\Omega / \omega_{0}$.

In [1] it was shown, that solutions obtained by Harmonic Balance or perturbation analysis in the range of low excitation frequencies can be identified being artifacts. Considering the Harmonic Balance method for the solution of $(2), x(\tau)$ is represented by the truncated Fourier series

$$
x(\tau)=\sum_{k=1}^{n}\left(a_{k} \cos (k \eta \tau)+b_{k} \sin (k \eta \tau)\right) .
$$


Introducing this in the Duffing equation (2) and expansion as Fourier series yields

$$
\sum_{k=1}^{n}\left(\tilde{a}_{k} \cos (k \eta \tau)+\tilde{b}_{k} \sin (k \eta \tau)\right)=f \cos (\eta \tau)-\sum_{k=n+1}^{3 n}\left(\tilde{a}_{k} \cos (k \eta \tau)+\tilde{b}_{k} \sin (k \eta \tau)\right)
$$

where the coefficients $\tilde{a}_{k}, \tilde{b}_{k}$ are nonlinear functions of the original ansatz coefficients $a_{k}, b_{k}$. Following Harmonic Balance, the higher order frequency terms

$$
\sum_{k=n+1}^{3 n}\left(\tilde{a}_{k} \cos (k \eta \tau)+\tilde{b}_{k} \sin (k \eta \tau)\right)
$$

in (4) are neglected and the coefficients $a_{k}, b_{k}$ are calculated from the thereby modified equation (4). In [1] the neglected terms (5) are used as a measure for the approximation error. These terms can be calculated from the found solution $a_{k}, b_{k}$. As an error it was chosen

$$
\hat{e}=\max _{0 \leq \tau \leq \frac{2 \pi}{\eta(n+1)}}\left\{\sum_{k=n+1}^{3 n}\left(\tilde{a}_{k} \cos (k \eta \tau)+\tilde{b}_{k} \sin (k \eta \tau)\right)\right\} .
$$

In the meanwhile we found, that a similar error criterion (and other ones) was also used in [5] and in referring work e.g. [6], [7]. It is important to be aware that this term assesses how well a found solution satisfies the differential equation and not how large the distance to an exact solution is. Therefore a small value of the error $\hat{e}$ does not necessarily imply that the found solution has converged to the exact solution as it is also discussed in [7]. Additionally, for finding potential additional solutions, which were not found with the Harmonic Balance ansatz (3), a refined perturbation analysis for low excitation frequencies was used in [1]. Therefore $\tau$ was considered to be

$$
\tau=\Omega t .
$$

Considering small excitation frequencies, the frequency ratio $\eta$ can then be considered to be small and is therefore replaced by $\varepsilon=\eta$. With this the equation

$$
\varepsilon^{2} x^{\prime \prime}(\tau)+\varepsilon 2 D x^{\prime}(\tau)+x(\tau)+\beta x^{3}(\tau)=\varepsilon \hat{f} \cos (\tau)
$$

with $\beta=\alpha /\left(m \omega_{0}^{2}\right)$ being negative and $f=\varepsilon \hat{f}$ is considered. Performing perturbation analysis the following solutions were obtained in [1]

$$
x_{01}(\tau)=-\sqrt{-\frac{1}{\beta}}-\frac{1}{2} f \cos (\tau), x_{02}(\tau)=f \cos (\tau), x_{03}(\tau)=\sqrt{-\frac{1}{\beta}}-\frac{1}{2} f \cos (\tau) .
$$

Hereby, the solutions $x_{01}$ and $x_{03}$ are vibrations about nonzero (unstable) equilibrium positions. The highest derivative term in equation (8) being of order $\varepsilon^{2}$ ("singular perturbation analysis") does in that case not cause any problems as only forced vibrations (and not free) are considered. [1]

Based on this a refined Harmonic Balance ansatz for solving the original equation (2) is used in

$$
x(\tau)=a_{0}+\sum_{k=1}^{n}\left(a_{k} \cos (k \eta \tau)+b_{k} \sin (k \eta \tau)\right)
$$

by adding a constant $a_{0}$. Corresponding results taken from [1] are shown in Figure 1, with the following parameters in equation (2)

$$
D=0.06, \varepsilon=-0.1 \text { and } f=0.2 .
$$

These results are presented with their absolut value, i.e. (3) represents the two solutions $x_{01}$ and $x_{03}$ from (9). From the error analysis performed in [1] the solutions (1) (with zero mean value) and (4) (with non-zero mean value) can be identified as artifacts while (2) represents the well known zero mean solution of the Duffing oscillator. This is an important result as the artifact result of type (1) can be found in many textbooks on nonlinear dynamics. Considering them as an existing 

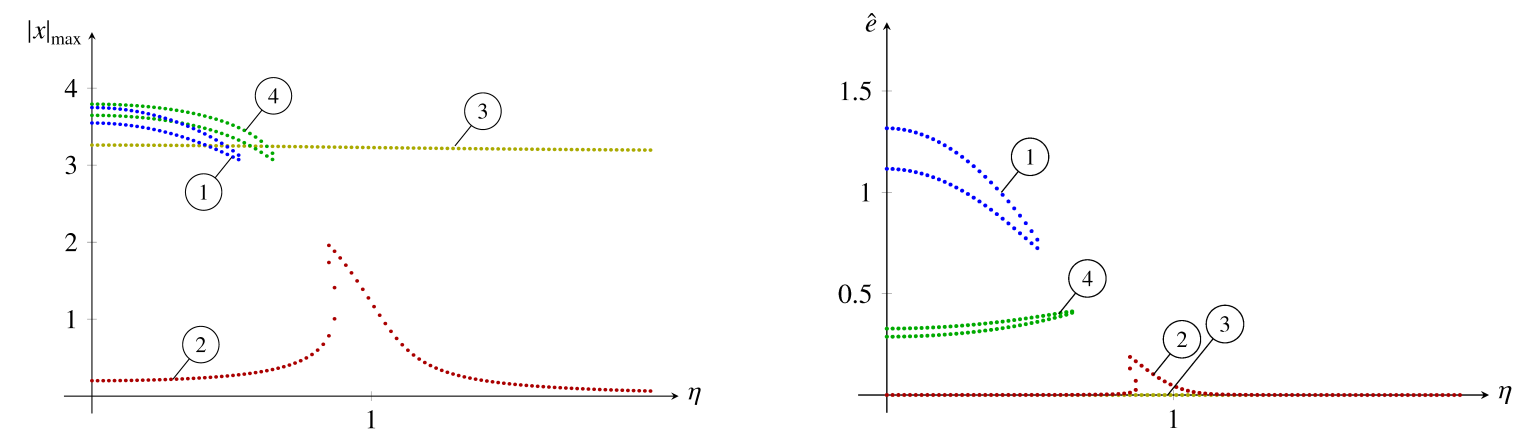

Fig. 1 Maximum displacements $|x|_{\max }$ and errors $\hat{e}$ for different types of solutions with refined Harmonic Balance according to (10) for $n=1[1]$

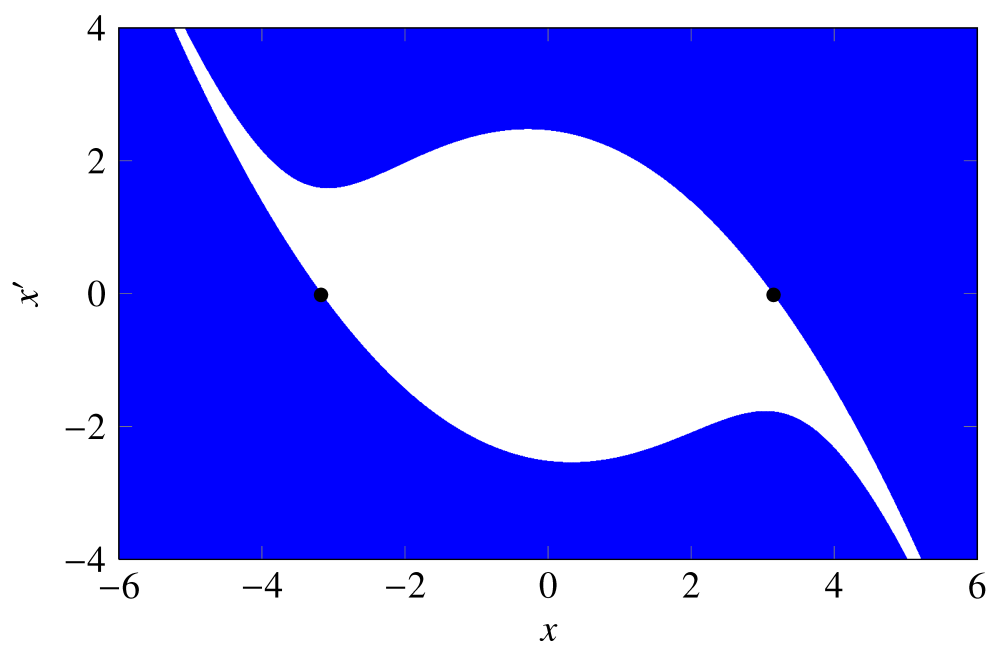

Fig. 2 Basins of attraction for $\eta=0.02$. White points correspond to initial conditions at $\tau=0$ leading to the solution (2) in Figure 1, blue points denote initial conditions for unbounded solutions drifting to plus or minus infinity. The two black points denote displacement and velocity for $\tau=0$ of solutions (3) in Figure 1 i.e. $x_{01}$ and $x_{03}$ from equation (9). Results from [1].

physical solution, stability analysis are also performed in several textbooks, where e.g. in [8] a Kryloff Bogoljuboff method is used therefore. Usually in these cases the result is, that the upper branch of the "nose" solution is stable, and the lower branch is unstable [2]. It shall also be mentioned, that there are examples, where applying such an error criterion may result at the right end of the nose of type (1) to low errors and therefore existing (and even stable) solutions, see [6].

Finally for this repetition from [1] the stability of the solutions (3) shall be discussed by Figure 2 where solutions resulting from the respective initial conditions are plotted as follows: White points correspond to initial conditions at $\tau=0$ leading to the solution (2) in Figure 1, while blue points denote initial conditions for unbounded solutions drifting to plus or minus infinity. The two black points denote displacement and velocity for $\tau=0$ of solutions (3) in Figure 1 i.e. $x_{01}$ and $x_{03}$ from equation (9). As these two solutions are on the borderline of the two solution types marked by blue and white it can be concluded that these solutions are unstable.

Based on these methods and results from [1] in the upcoming sections the following results are presented. The next section will contain a stability analysis of the found solutions performed by the method of slowly changing phase and amplitude. It is remarkable that this analysis will also lead to a wrong result, i.e. also will produce an artifact. In the next section an extended Duffing oscillator with additional nonlinear terms is investigated producing "island"-like solutions in classical Harmonic Balance. Using the described methods, it is investigated, whether these solutions are artifacts or not. 
3 Artifacts in stability analysis by the method of slowly changing phase and amplitude

In the following, the solution $x_{03}$ in (9) shall be recalculated and the stability be investigated by using the method of slowly changing phase and amplitude. Therefore, in equation (8) the transformation

$$
x(\tau)=\sqrt{-\frac{1}{\beta}}+y(\tau)
$$

is introduced. This results in the nonlinear differential equation for $y(\tau)$

$$
\varepsilon^{2} y^{\prime \prime}(\tau)+\varepsilon 2 D y^{\prime}(\tau)-2 y(\tau)+3 \gamma y^{2}(\tau)-\gamma^{2} y^{3}(\tau)=\varepsilon \hat{f} \cos (\tau) .
$$

with $\gamma=\sqrt{-\beta}$. Following the method of slowly changing phase and amplitude $y$ and $y^{\prime}$ are replaced by amplitude $a$ and phase $\psi$ by

$$
y=a \cos (\tau+\psi) ; \quad y^{\prime}=-a \sin (\tau+\psi)
$$

respectively

$$
a=\sqrt{y^{2}+{y^{\prime}}^{2}} ; \quad \psi=\arctan \left(-\frac{y^{\prime}}{y}\right)-\tau
$$

taking into consideration, that the excitation is proportional to $\cos \tau$. Performing the coordinate transformation to phase and amplitude results in

$$
\begin{aligned}
\psi^{\prime}= & -1+\sin ^{2}(\tau+\psi)-\frac{2 D}{\varepsilon} \sin (\tau+\psi) \cos (\tau+\psi)-\frac{2}{\varepsilon^{2}} \cos ^{2}(\tau+\psi) \\
& +\frac{3 \gamma a}{\varepsilon^{2}} \cos ^{3}(\tau+\psi)-\frac{\gamma^{2} a^{2}}{\varepsilon^{2}} \cos ^{4}(\tau+\psi)-\frac{\hat{f}}{\varepsilon a} \cos (\tau+\psi) \cos \tau
\end{aligned}
$$

and

$$
\begin{aligned}
a^{\prime}= & a\left(-1-\frac{2}{\varepsilon^{2}}\right) \sin (\tau+\psi) \cos (\tau+\psi)-\frac{2 D a}{\varepsilon} \sin ^{2}(\tau+\psi)+\frac{3 \gamma a^{2}}{\varepsilon^{2}} \cos ^{2}(\tau+\psi) \sin (\tau+\psi) \\
& -\frac{\gamma^{2} a^{3}}{\varepsilon^{2}} \cos ^{3}(\tau+\psi) \sin (\tau+\psi)-\frac{\hat{f}}{\varepsilon} \cos \tau \sin (\tau+\psi) .
\end{aligned}
$$

Up to this point, the calculation is exact. Following the method of slowly changing phase and amplitude, the right hand side of equations (16) and (17) is averaged in that way that the integral $\frac{1}{2 \pi} \int_{0}^{2 \pi}(\ldots) \mathrm{d} \tau$ is calculated. This results in the simplified equations

$$
\begin{gathered}
\psi^{\prime}=-\frac{1}{\varepsilon^{2}}-\frac{1}{2}-\frac{3}{8} \frac{\gamma^{2} a^{2}}{\varepsilon^{2}}-\frac{\hat{f}}{2 \varepsilon a} \cos \psi \\
a^{\prime}=-\frac{D a}{\varepsilon}-\frac{1}{2} \frac{\hat{f}}{\varepsilon} \sin \psi
\end{gathered}
$$

From equations (18) and (19) the stationary solution, i.e. a solution with constant phase and amplitude $\psi^{\prime}=0, a^{\prime}=0$ can be calculated. Considering only the lowest order terms in $\varepsilon$ and linearizing gives the results

or

$$
a=-\frac{1}{2} \varepsilon \hat{f}=-\frac{1}{2} f, \quad \psi=0
$$

$$
a=\frac{1}{2} \varepsilon \hat{f}=\frac{1}{2} f, \quad \psi=\pi
$$

i.e. in both cases the same result as obtained as the the zeroth approximation $x_{03}$ by the perturbation analysis in (9). Using the parameters from (11) and the frequency ratio $\eta=\varepsilon=0.1$, the tool Mathematica in fact provides numerically the real solutions of (18) and (19) as $a=0.0961809, \psi=$ 0.00577088 or $a=-0.0961809, \psi=3.13582$, which is very close to the approximate solutions (20) and $(21)(f / 2=0.1$ in this case $)$. 

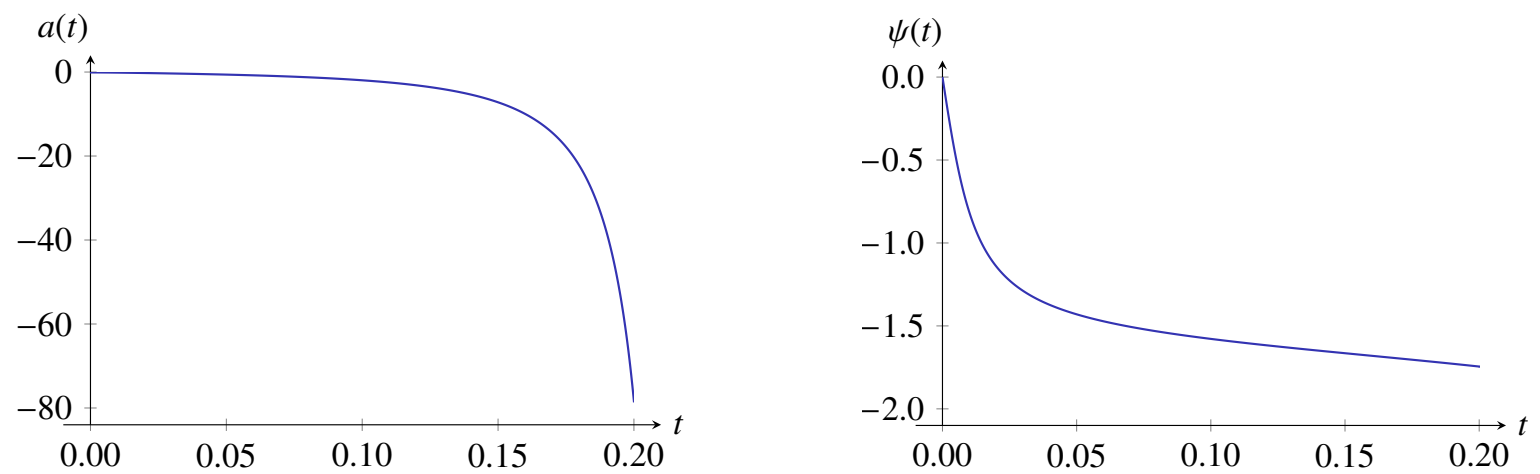

Fig. 3 Numerical integration of equations (16) and (17) with initial conditions $a(0)=-0.1, \psi=0$.
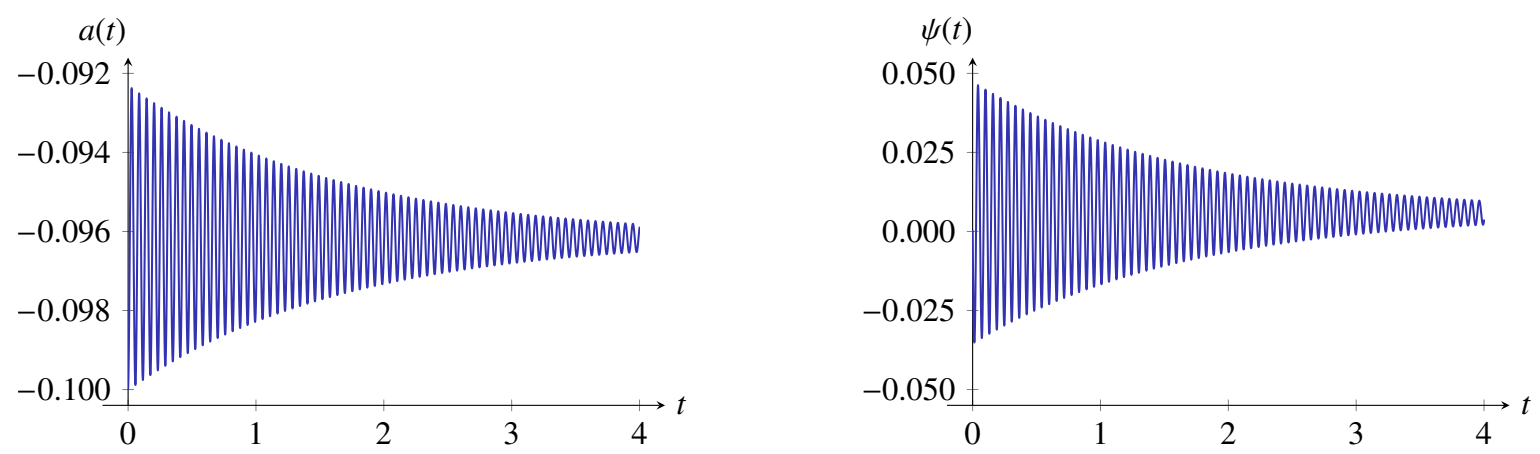

Fig. 4 Numerical integration of equations (18) and (19) with initial conditions $a(0)=-0.1, \psi=0$.

While the stationary solution of the approximation by slowly changing phase and amplitude is very close to the one calculated by Harmonic Balance or perturbation analysis this does not hold for the found stability behavior. In fact it was shown in section 2 and especially figure 2 that this solution is unstable. This point shall be first investigated by some results of numerical integration in Figures 3 and 4 .

Results in Figure 3 clearly shows, that we have a solution drifting away. The instability of the solution (9) can be clearly seen also here. This changes, if we now consider the result of the numerical integration of equations (18) and (19) as displayed in Figure 4. Here the solution converges approximately to the stationary solution (20), i.e. the solution shows an asymptotically stable behavior for the same initial conditions.

A corresponding systematic stability analysis shall now be performed based on the averaged equations (18) and (19). Linearizing with respect to the solution (20) with deviations $\Delta \psi, \Delta a$ from this solution gives an equation of the form

$$
\begin{aligned}
\left(\begin{array}{c}
\Delta \psi^{\prime} \\
\Delta a^{\prime}
\end{array}\right) & =\left(\begin{array}{cc}
0 & \frac{3}{8} \frac{\hat{f} \gamma^{2}}{\varepsilon}+\frac{2}{\hat{f} \varepsilon^{3}} \\
-\frac{1}{2} \frac{\hat{f}}{\varepsilon} & -\frac{D}{\varepsilon}
\end{array}\right)\left(\begin{array}{c}
\Delta \psi \\
\Delta a
\end{array}\right)+\left(\begin{array}{c}
-\frac{1}{2}+\frac{3}{24} \gamma^{2} \hat{f}^{2} \\
0
\end{array}\right) \quad \text { or } \\
\mathbf{r}^{\prime} & =\mathbf{A r}+\mathbf{v}
\end{aligned}
$$

where the constant vector $\mathbf{v}$ results from the fact, that (20) is only an approximate solution of the equations (18) and (19). Analyzing matrix A now for their eigenvalues gives the result observed already in numerical integration, that (20) is asymptotically stable! This result is obviously wrong and an artifact of the method of slowly changing phase and amplitude!

In fact the same effect can be already observed, when a simple linear undamped oscillator with negative restoring is considered:

$$
y^{\prime \prime}-y=f \cos \tau \text {. }
$$


Conducting the transformation (14) results in the equations for phase and amplitude

$$
\begin{aligned}
& \psi^{\prime}=-1+\sin ^{2}(\tau+\psi)-\cos ^{2}(\tau+\psi)-\frac{f}{a} \cos (\tau+\psi) \cos \tau, \\
& a^{\prime}=-2 a \sin (\tau+\psi) \cos (\tau+\psi)-f \sin (\tau+\psi) \cos \tau .
\end{aligned}
$$

Performing again the averaging by calculating the integrals $\frac{1}{2 \pi} \int_{0}^{2 \pi}(\ldots) \mathrm{d} \tau$ of the right hand sides result in

$$
\begin{aligned}
\psi^{\prime} & =-1-\frac{1}{2} \frac{f}{a} \cos \psi, \\
a^{\prime} & =-\frac{1}{2} f \sin \psi
\end{aligned}
$$

Again from this the stationary solution, i.e. the solution with constant phase and amplitude $\psi^{\prime}=$ $0, a^{\prime}=0$ can be calculated. This results in

$$
a=-\frac{1}{2} f, \quad \psi=0
$$

which is in fact an exact (particular) solution of the original equation (24). Obviously this solution is unstable, as can be easily checked by considering the original linear equation. Linearizing now equations (27) and (28) with respect to (29) results in

$$
\begin{array}{rlr}
\left(\begin{array}{c}
\Delta \psi^{\prime} \\
\Delta a^{\prime}
\end{array}\right) & =\left(\begin{array}{cc}
0 & \frac{2}{f} \\
-\frac{1}{2} f & 0
\end{array}\right)\left(\begin{array}{l}
\Delta \psi \\
\Delta a
\end{array}\right) \quad \text { or } \\
\mathbf{r}^{\prime} & =\mathbf{A r}
\end{array}
$$

and via an eigenvalue analysis it can be seen, that the solution (29) should be stable! Again the method of slowly changing phase and amplitude fails here in case of negative restoring with respect to stability analysis. For both examples considered in this section the stationary solution is exact in the linear case or matches with perturbation analysis in the nonlinear case respectively while the transient behavior is qualitatively wrong! At this point it should be remembered, how such methods are usually introduced, e.g. in [3] oder [9]. Herein an equation of type

$$
\ddot{x}+\omega_{0}^{2} x=f(x, \dot{x})
$$

i.e. with positive restoring is considered, with the assumption of a small right hand side. In fact, the basic assumption of "slowly changing phase and amplitude" is violated in the presence case of an unstable solution.

\section{Modified Duffing oscillator with "island" solutions}

In the following a modified Duffing oscillator will be investigated with the methods introduced in section 2 . The corresponding equation of motion is given by

$$
x^{\prime \prime}+2 D x^{\prime}+x+\varepsilon_{1} x^{3}+\varepsilon_{2} x^{2} x^{\prime}=f_{1} \cos (\eta \tau)+f_{2} x^{2} \cos (\eta \tau) .
$$

where the same denominations are used as in (2). Herein the classic Duffing Oscillator with the cubic nonlinearity with parameter $\varepsilon_{1}$ is complemented with a cubic damping term $x^{2} x^{\prime}$ with parameter $\varepsilon_{2}$ and the harmonic excitation with intensity $f_{1}$ is supplemented by a nonlinear parameter excitation term with constant $f_{2}$. Following e.g. [10] such an equation can be obtained, when piezoceramic continua are modeled with nonlinear as well conservative as non-conservative additions in their constitutive equations with respect to elastic and electro-mechanical coupling terms. This does not mean, that the parameter chosen here represent a real piezoceramic. Depending on the chosen parameters there may be a variety of different solutions including amplitude/frequency regions with solutions separated from other solutions. Those solutions are in the following called as in some literature, e.g. 
[11], solution "islands". In the following, the equation (33) is considered for several parameter sets and the solutions are subsequently investigated for artifacts with the method described in section 2 .

Equation (33) is solved approximately by again using the Harmonic Balance ansatz

$$
x(\tau)=\sum_{k=1}^{n}\left(a_{k} \cos (k \eta \tau)+b_{k} \sin (k \eta \tau)\right) .
$$

While in [1] special attention was given to solutions which contain an additional constant term $a_{0}$, this is neglected in the actual case as we want to focus on a new type of solutions, the "island" solutions which have zero mean values.

Introducing (34) into (33) and neglecting higher order terms results in a system of nonlinear algebraic equations for the $a_{k}$ and $b_{k}$. From this, in order to visualize the solutions from Harmonic Balance, $\hat{x}$ is defined as

$$
\hat{x}=\max _{0 \leq \tau \leq \frac{2 \pi}{\eta}}\left\{\sum_{k=1}^{n}\left(a_{k} \cos (k \eta \tau)+b_{k} \sin (k \eta \tau)\right)\right\} .
$$

As in the earlier case of the "pure" Duffing oscillator, the coefficients $\tilde{a}_{k}, \tilde{b}_{k}$ of the neglected higher oder terms can be calculated having obtained a solution for $a_{k}, b_{k}$ and an error $\hat{e}$ is defined from this by

$$
\hat{e}=\max _{0 \leq \tau \leq \frac{2 \pi}{\eta}}\left\{\sum_{k=n+1}^{3 n}\left(\tilde{a}_{k} \cos (k \eta \tau)+\tilde{b}_{k} \sin (k \eta \tau)\right)\right\} .
$$

Finally a relative error $\tilde{e}$ is introduced as

$$
\tilde{e}=\frac{\hat{e}}{\hat{x}}
$$
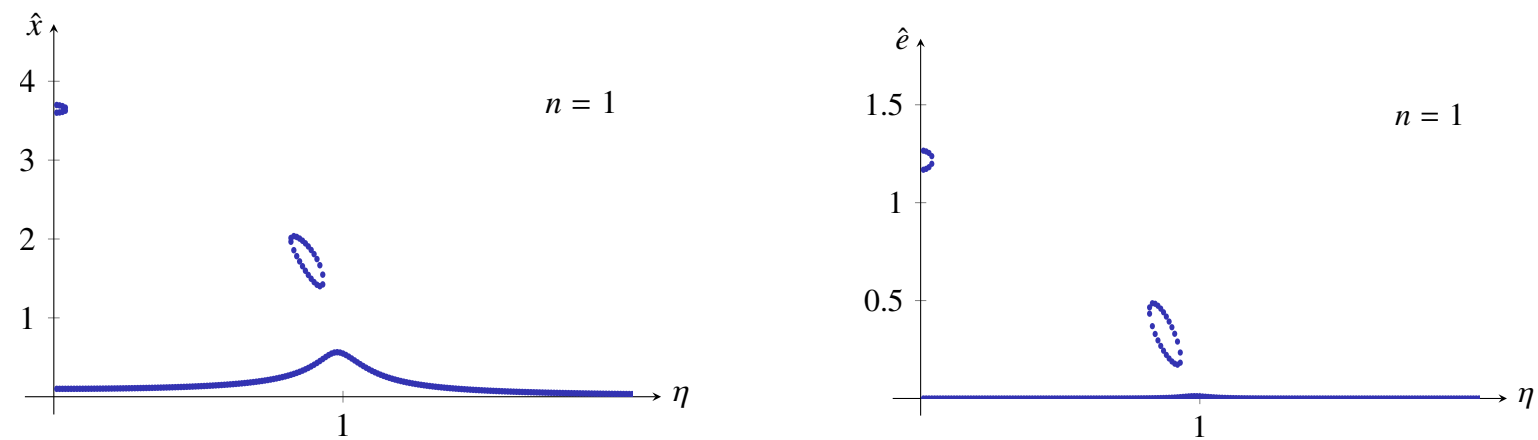

Fig. 5 Solutions for the extended Duffing oscillator (33) with Harmonic Balance ansatz (34) in case of $n=1$. $\hat{x}$ according to (35) (left) and error $\hat{e}$ according to (36) (right). Parameter: $\varepsilon_{1}=-0.1, \varepsilon_{2}=-0.25, D=0.1$, $f_{1}=0.1$ and $f_{2}=0$.

Figures 5 and 6 show corresponding results in the case of $n=1$ in ansatz (34) for the parameter $\varepsilon_{1}=-0.1, \varepsilon_{2}=-0.25, D=0.1, f_{1}=0.1$ and $f_{2}=0$. It can be observed that additional to the resonance peak and the "nose"-like solution, there is an additional solution "island" in the frequency range between the two aforementioned solutions (Figure 5 (left)). Based on the results from before, it can be expected, that the "nose" solution is an artifact but what is about the "island" solution? In fact the error analysis (Figure 5 (right)) shows, that the error $\hat{e}$ is somewhat large in that case, but lower than in the case of the "nose" solution. This is also confirmed, when the relative error $\tilde{e}$ (Figure 6 (right)) is considered. Finally introducing an error limit of $1 \%$ for $\tilde{e}$ results in the color code in Figure 6 (left) highlighting that also in the resonance peak the error is somewhat high. With blue, solutions with $\tilde{e}$ lower than $1 \%$ are marked while red color denotes solutions with larger relative error. 

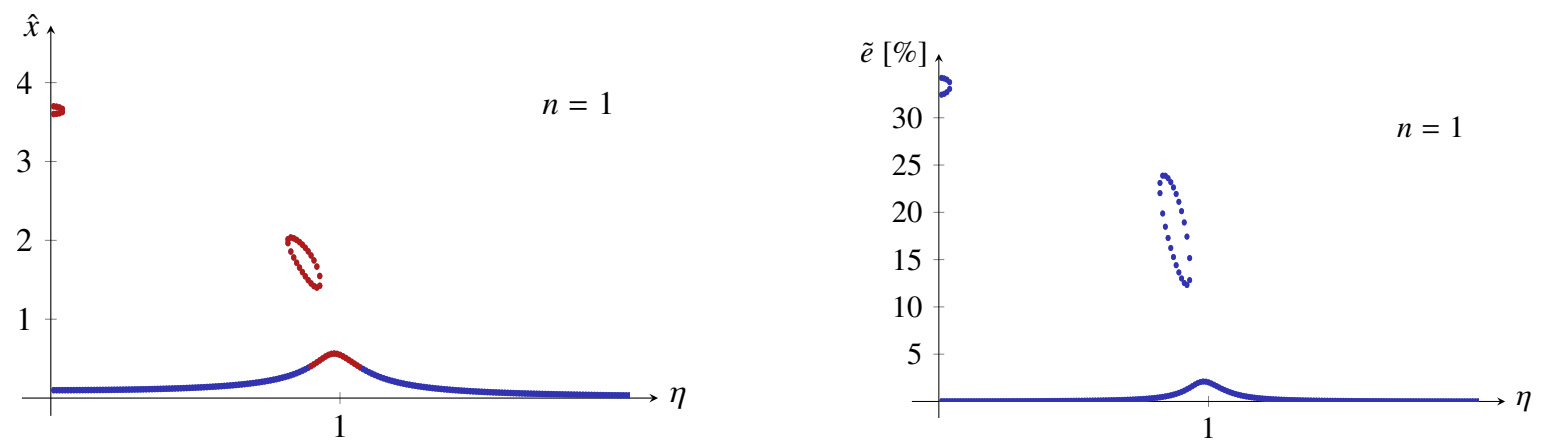

Fig. 6 Solutions for the extended Duffing oscillator (33) with Harmonic Balance ansatz (34) in case of $n=1$. $\hat{x}$ according to (35) (left), where solutions with relative error larger than $1 \%$ are marked with red and lower than $1 \%$ by blue color. Relative error $\tilde{e}$ according to (37)(right). Parameter: $\varepsilon_{1}=-0.1, \varepsilon_{2}=-0.25, D=0.1$, $f_{1}=0.1$ and $f_{2}=0$.
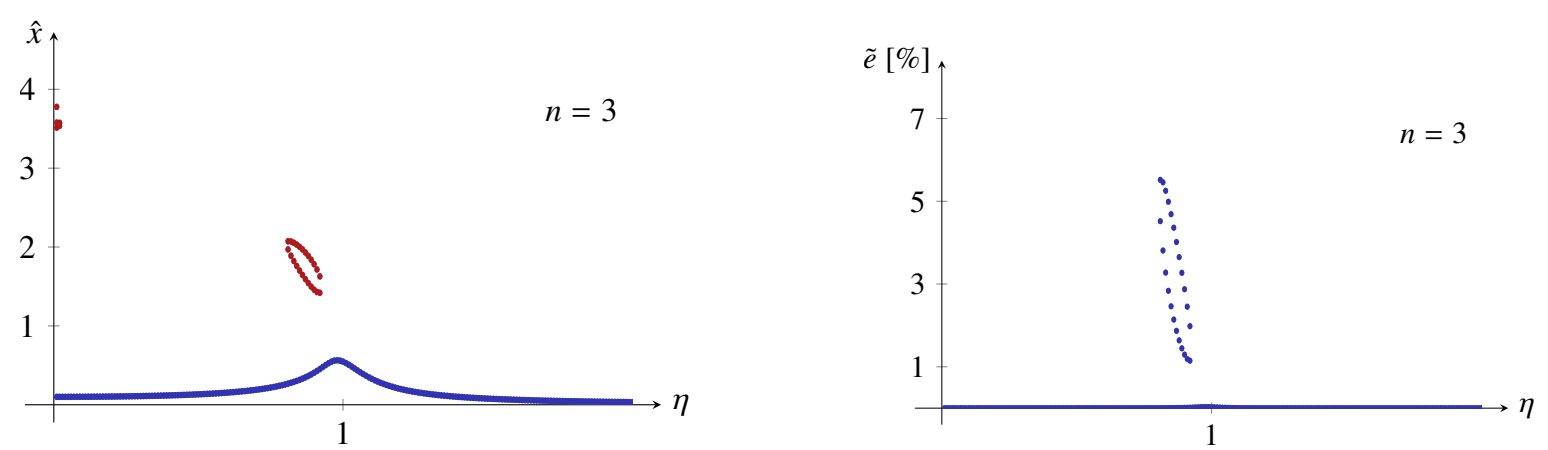

Fig. 7 Solutions for the extended Duffing oscillator (33) with Harmonic Balance ansatz (34) in case of $n=3$. $\hat{x}$ according to (35)(left) and error $\tilde{e}$ according to (37) (right). For $\hat{x}$ red denotes solutions with large errors and blue solutions with low errors. Parameter: $\varepsilon_{1}=-0.1, \varepsilon_{2}=-0.25, D=0.1, f_{1}=0.1$ and $f_{2}=0$.
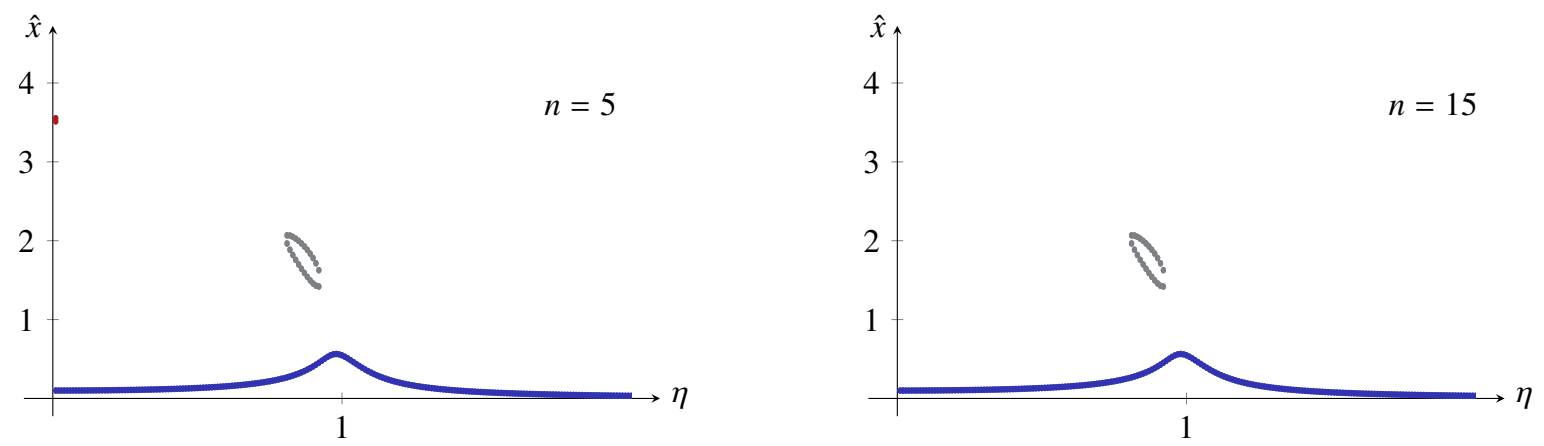

Fig. 8 Solutions for extended Duffing oscillator (33) with Harmonic Balance ansatz (34) in case of $n=5$ (left) and $n=15$ (right). $\hat{x}$ according to (35) where solutions with relative error larger than $1 \%$ are marked with red color. Solutions with relative error lower than $1 \%$ are marked by blue color in case of stable and grey color in case of unstable ones. Parameter: $\varepsilon_{1}=-0.1, \varepsilon_{2}=-0.25, D=0.1, f_{1}=0.1$ and $f_{2}=0$.

In the following, the ansatz order is increased to $n=3$. Figure 7 shows corresponding results for $\hat{x}$ (left) and $\tilde{e}$ (right). The results doesn't change qualitatively but there is a remarkable decrease of the error of the "island" solution. Nevertheless relative errors $\tilde{e}$ are still larger than $1 \%$ in the "island" solution as can be seen by red color of this solution in Figure 7 (left). If the ansatz order is increased to $n=5$ the relative error of the "island" solution further decreases and becomes lower than 1\%. This is displayed in Figure 8 (left). Solutions with $\tilde{e}$ lower than $1 \%$ are now also checked for their stability by introducing them into the original equation and linearizing with respect to this solution. This results in a linear second order differential equation with time dependent coefficients 

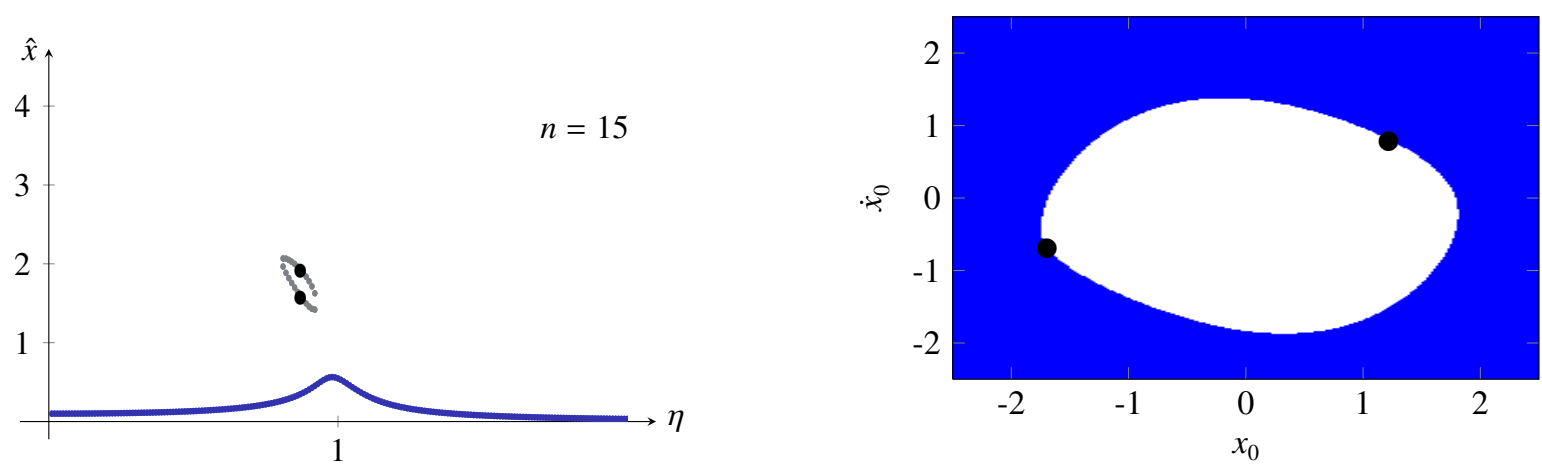

Fig. 9 Left: same result as in Figure 8 but with marked "island" solutions at $\eta=0.87$. Right: Basins of attraction for $\eta=0.87$. White points correspond to initial conditions at $\tau=0$ leading to the regular stable solution, blue points denote initial conditions for unbounded solutions drifting to plus or minus infinity. The two black points denote displacement and velocity for $\tau=0$ for the two solutions marked on the left side.

whose trivial solution is checked for stability by Floquet theory. Unstable low error solutions are marked with grey color while blue is used for stable solutions. Increasing the ansatz order to $n=15$ doesn't show any more dramatic changes in this behavior with the final result, that the "island" solution seems to exist but is unstable and the "nose"-like solution vanishes completely for higher ansatz orders and is therefore an artifact. This result is shown in Figure 8 (right). This can be also seen in Figure 9 where on the left hand side the two "island" solutions are marked for $\eta=0.87$. On the right hand it can be clearly seen, that these two solutions are located on the separatrix separating basins of attraction of the stable regular solution and the solutions drifting to plus or minus infinity.

In the following a slightly different parameter set is investigated namely $\varepsilon_{1}=-0.1, \varepsilon_{2}=-0.2$, $D=0.1, f_{1}=0.1$ and $f_{2}=0.1$ which shows in parts a different behavior. Only the figures displaying $\hat{x}$ using the color code: red are solutions with relative error larger than $1 \%$, grey are low error unstable solutions and blue are low error stable solutions are shown.
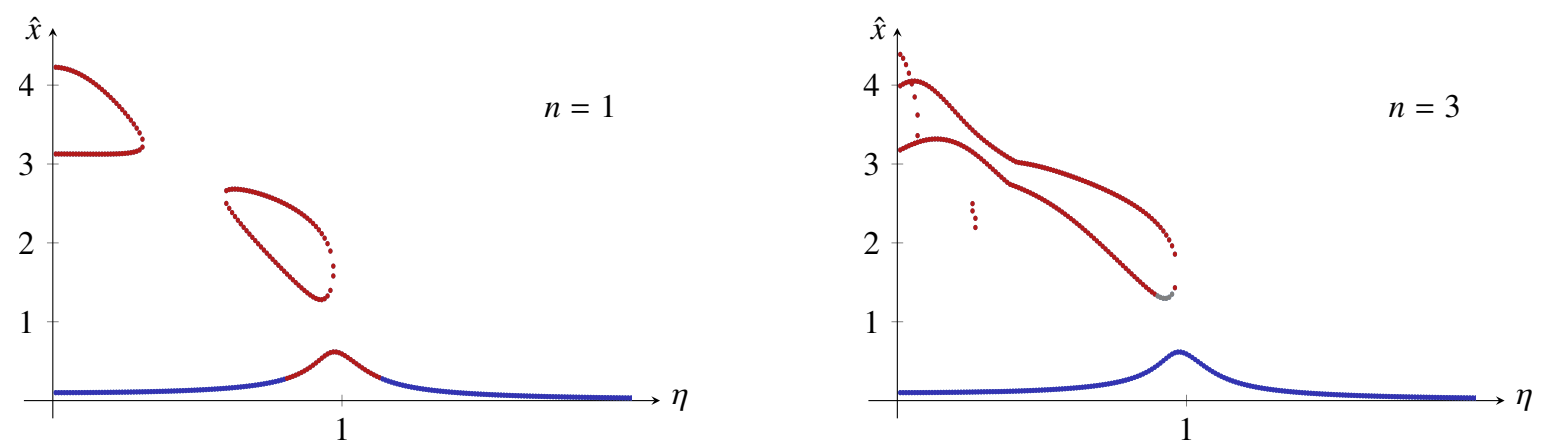

Fig. 10 Solutions $\hat{x}$ for the extended Duffing oscillator (33) with Harmonic Balance ansatz (34) in case of $n=1$ (left) and $n=3$ (right). Red denotes solutions with large errors, grey unstable solutions with low errors and blue stable solutions with low errors. Parameter: $\varepsilon_{1}=-0.1, \varepsilon_{2}=-0.2, D=0.1, f_{1}=0.1$ and $f_{2}=0.1$.

As can be seen in Figure 10 (left) for ansatz order $n=1$ again a "nose"-like and an "island" solution occurs. The case $n=3$ in Figure 10 (right) shows that these two solutions merge for this higher ansatz order. Again for the higher ansatz orders $n=9$ and $n=13$ parts of this new "island" solution have small errors and are in general unstable, but there is also a small range of frequencies close to $\eta=1$ where there is a stable solution. Compared to other parameter results considered in the following, the used method shows a comparably slow convergence of the shape and the characteristics of the solutions. Therefore also higher ansatz numbers up to $n=59$ are considered. Corresponding results are shown in Figure 12 for $n=49$ and $n=59$. Here it can be seen that aside the left end of the former "nose" and "island" there exist unstable solutions. 

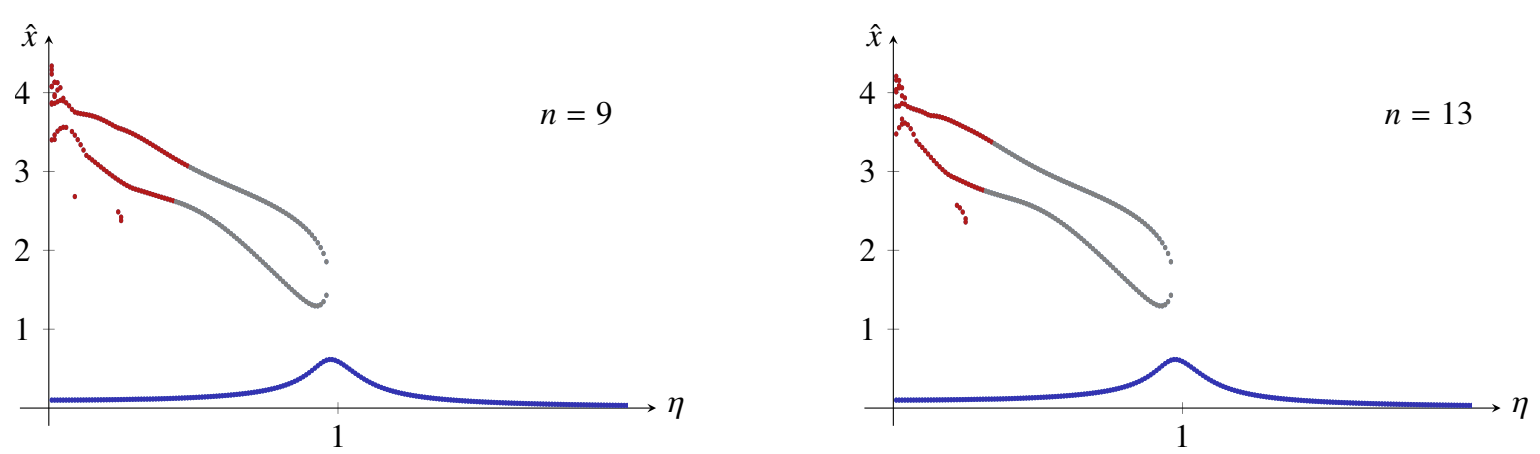

Fig. 11 Solutions $\hat{x}$ for the extended Duffing oscillator (33) with Harmonic Balance ansatz (34) in case of $n=9$ (left) and $n=13$ (right). Red denotes solutions with large errors, grey unstable solutions with low errors and blue stable solutions with low errors. Parameter: $\varepsilon_{1}=-0.1, \varepsilon_{2}=-0.2, D=0.1, f_{1}=0.1$ and $f_{2}=0.1$.
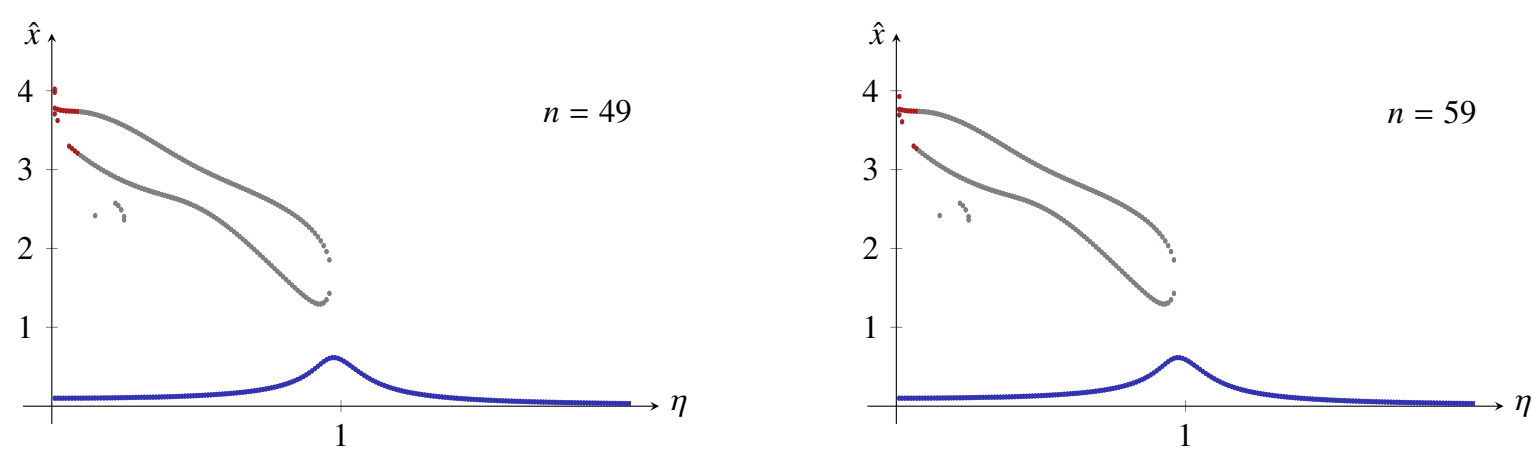

Fig. 12 Solutions $\hat{x}$ for the extended Duffing oscillator (33) with Harmonic Balance ansatz (34) in case of $n=49$ (left) and $n=59$ (right). Red denotes solutions with large errors, grey unstable solutions with low errors and blue stable solutions with low errors. Parameter: $\varepsilon_{1}=-0.1, \varepsilon_{2}=-0.2, D=0.1, f_{1}=0.1$ and $f_{2}=0.1$.

Coming back to convergence of the results our experience so far is, that in regions with artifacts (large relative errors), the solutions change their shape with increasing ansatz order and the coefficients $a_{k}$ and $b_{k}$ do not converge. On the other hand, low relative errors go along with rapidly converging $a_{k}$ and $b_{k}$ in the Harmonic Balance ansatz (34). The solutions with high ansatz order also confirm that the solutions showing low errors in low ansatz orders are reliable. On the other hand for regions of solutions showing large errors in low ansatz orders higher orders must be calculated in order to get a true picture of the behavior.

Finally two other parameter sets shall be considered. As for these parameter sets the proposed method shows a much faster convergence with respect to shape and characteristic of the solutions, the ansatz orders are limited to lower numbers than in the example before. While the two parameter sets looked on so far show both a softening characteristic together with an "island" solution we will in the following look first on a stiffening case. The corresponding parameter set is $\varepsilon_{1}=0.1, \varepsilon_{2}=-0.25$, $D=0.1, f_{1}=0.1$ and $f_{2}=0.1$. The results are shown in Figures 13 and 14 for ansatz dimensions $n=1,3,5$ and 7 . Also in this case, a solution "island" can be observed. This solution remains almost unchanged when increasing the ansatz order but the error can significantly be reduced with the final result, that the "island" solution seems to exist, but is unstable.

The next parameter set is $\varepsilon_{1}=0.0, \varepsilon_{2}=-0.2, D=0.1, f_{1}=0.1$ and $f_{2}=0.1$ where the vanishing $\varepsilon_{1}$ is resulting in neither a stiffening nor a softening characteristic. The results are shown in Figures 15 and 16 for ansatz dimensions $n=1,3,5$ and 7. Also in this case an "island" solution exist and the increasing order in Harmonic Balance again result in a decreasing error. Parts of the "island" solution are determined for having small error and being stable (blue color) in case of ansatz order $n=3$ or 5 . In fact the result for $n=7$ (Figure 16 (right)) shows, that the complete "island" solution is unstable. 

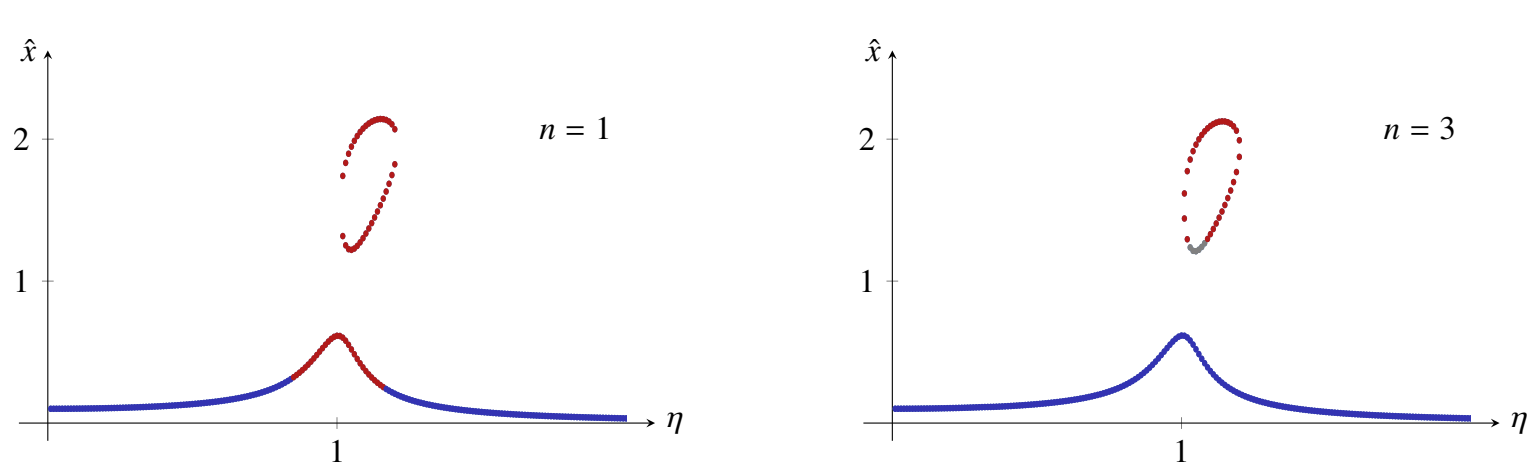

Fig. 13 Solutions $\hat{x}$ for the extended Duffing oscillator (33) with Harmonic Balance ansatz (34) in case of $n=1$ (left) and $n=3$ (right). Red denotes solutions with large errors, grey unstable solutions with low errors and blue stable solutions with low errors. Parameter: $\varepsilon_{1}=0.1, \varepsilon_{2}=-0.25, D=0.1, f_{1}=0.1$ and $f_{2}=0.1$.
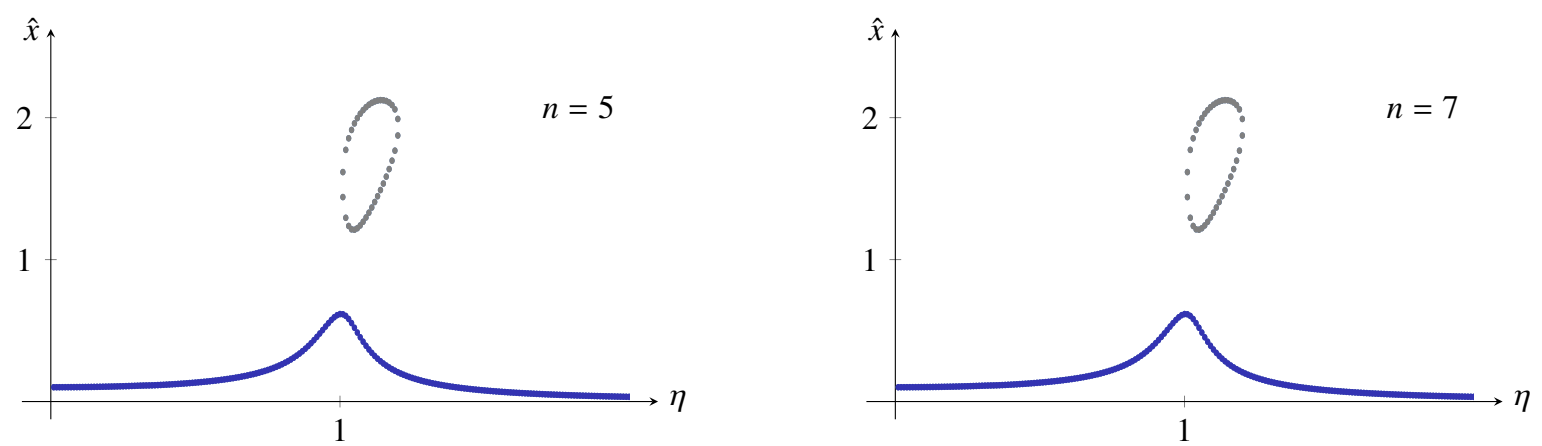

Fig. 14 Solutions $\hat{x}$ for the extended Duffing oscillator (33) with Harmonic Balance ansatz (34) in case of $n=5$ (left) and $n=7$ (right). Red denotes solutions with large errors, grey unstable solutions with low errors and blue stable solutions with low errors. Parameter: $\varepsilon_{1}=0.1, \varepsilon_{2}=-0.25, D=0.1, f_{1}=0.1$ and $f_{2}=0.1$.
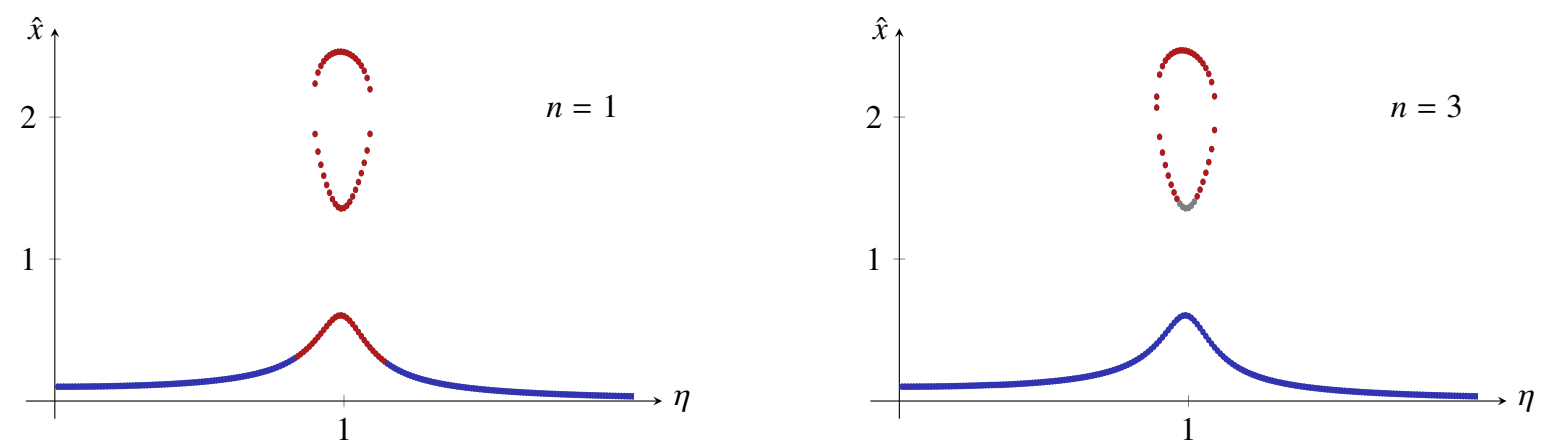

Fig. 15 Solutions $\hat{x}$ for the extended Duffing oscillator (33) with Harmonic Balance ansatz (34) in case of $n=1$ (left) and $n=3$ (right). Red denotes solutions with large errors, grey unstable solutions with low errors and blue stable solutions with low errors. Parameter: $\varepsilon_{1}=0.0, \varepsilon_{2}=-0.2, D=0.1, f_{1}=0.1$ and $f_{2}=0.1$.

\section{Conclusions}

In this paper we considered solutions for simple and modified Duffing oscillators obtained by the methods of Harmonic Balance and slowly changing phase and amplitude. The results were especially investigated with respect to potential artifacts. After an introduction of the basic method used in [1] using Harmonic Balance and an error definition first the method of slowly changing phase and amplitude is used to determine stability of non-zero mean solutions from a softening Duffing oscillator. It can be shown, that this method is resulting in a definitely wrong stability statement in this case due to the violation of the basic assumption, i.e. a slowly changing phase and amplitude which does not 

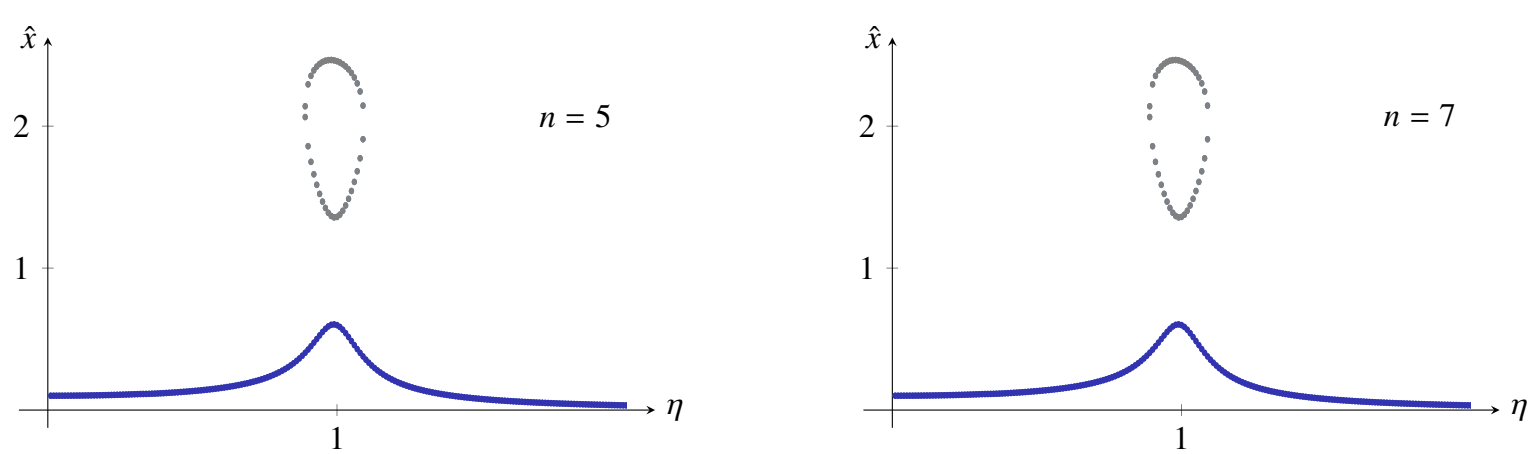

Fig. 16 Solutions $\hat{x}$ for the extended Duffing oscillator (33) with Harmonic Balance ansatz (34) in case of $n=5$ (left) and $n=7$ (right). Red denotes solutions with large errors, grey unstable solutions with low errors and blue stable solutions with low errors. Parameter: $\varepsilon_{1}=0.0, \varepsilon_{2}=-0.2, D=0.1, f_{1}=0.1$ and $f_{2}=0.1$.

hold any more in that case. Next, an extended Duffing oscillator adding a nonconservative cubic term and a nonlinear parametric excitation is investigated using Harmonic Balance and error criterion. This equation of motion shows additional separated new "island" solutions, which are considered for their error, and, if existing (i.e. small errors), for their stability. It could be shown, that the solution figure may change significantly when increasing the solution order and taking into account the error criterion.

Actually we are investigating further nonlinear dynamical systems with this method. For example the equation

$$
x^{\prime \prime}+2 D x^{\prime}+x+\varepsilon_{1} x^{3}+\varepsilon_{2} x^{\prime 3}\left(\tau-\tau_{0}\right)=f \cos (\eta \tau)
$$

where, as an additional nonlinearity, a cubic velocity term with a time shift $\tau^{\prime}$ is introduced. Such equations are considered in control and can be found e.g. in [12] or [13]. This equation also show "island" solutions, but stability is harder to determine due to the time shift. Additionally bistable piezo beam energy harvesting systems are investigated, where the discretized nonlinear beam equation(s) are coupled with a linear differential equation for the electric circuit. In that case, for higher ansatz orders, numerous solutions are found by Harmonic Balance. Following the procedures described in the present paper, artifacts and unstable solutions can be identified and a clear figure of possible (stable) solutions is derived.

\section{References}

1. von Wagner, U.; Lentz L. (2016) On some aspects of the dynamic behavior of the softening Duffing oscillator under harmonic excitation, Archive of Applied Mechanics 86 (8), 1383-1390.

2. Kovacic, I.; Brennan, M. (2011) The Duffing Equation: Nonlinear Oscillators and their Behaviour. John Wiley \& Sons, Ltd,

3. Hagedorn, P. (1978) Nichtlineare Schwingungen. Akademische Verlagsgesellschaft, Wiesbaden

4. Mook, D. T.; Nayfeh, A. H.(1979) Nonlinear Oscillations. Wiley, New York

5. Urabe, M.; Reiter, A. (1966) Numerical Computation of Nonlinear Forced Oscillations by Galerkin's Procedure. Journal of Mathematical Analysis and Applications 14, 107 - 140

6. van Dooren R. (1988) On the transition from regular to chaotic behaviour in the Duffing oscillator. Journal of Sound and Vibration 2, 123, 327-339.

7. Ferri A. A.; Leamy, M. J. (2009) Error Estimates for Harmonic-Balance Solutions of Nonlinear Dynamical Systems. Proceedings of 50th AIAA/ASME/ASCE/AHS/ASC Structures, Structural Dynamics, and Materials Conference 2009, AIAA 2009-2667

8. Kauderer, H. (1958) Nichtlineare Mechanik. Springer, Berlin

9. Bogoljubow N. N., Mitropolski, J. A. (1965) Asymptotische Methoden in der Theorie der nichtlinearen Schwingungen. Akademie Verlag, Berlin

10. Parashar Sandeep Kumar, von Wagner Utz, Hagedorn Peter (2005) Nonlinear shear-induced flexural vibrations of piezoceramic actuators: experiments and modeling. Journal of Sound and Vibration Volume 285, p. $989-1014$

11. Narimani A, Golnaraghi M F, Nakhaie Jazar G (2004) Sensitivity Analysis of the Frequency Response of a Piecewise Linear System in a Frequency Island. Journal of Vibration and Control Volume 10, p. 175-198

12. Gao X, Chen Q (2014) Nonlinear analysis, design and vibration isolation for a bilinear system with time delayed cubic velocity feedback. Journal of Sound and Vibration Volume 333, p. 1562 - 1576 
13. Wang Yong, Li Shunming, Cheng Chun, Jiang Xingxing (2015) Dynamic Analysis of a High-Static-LowDynamic-Stiffness Vibration Isolator with Time-Delayed Feedback Control. Shock and Vibration Volume 2015, Article ID 712851 